Stuhec Matej, ${ }^{12 *}$ Pharm.D., Ph.D., Serra-Mestres Jordi, ${ }^{3}$ L.M.S, M.R.C.Psych

\title{
Antidepressant drugs for older patients on polypharmacy: a systematic review reveals best evidence for sertraline
}

\author{
'Department for Clinical Pharmacy, Ormoz Psychiatric Hospital, Ormoz, Slovenia, European Union \\ ${ }^{2}$ Faculty of Pharmacy, University of Ljubljana, Ljubljana, Slovenia, European Union \\ ${ }^{3}$ Central and North West London NHS Foundation Trust, London, UK \\ *email: matejstuheclagmail.com
}

DOI: 10.2478/gp-2018-0005

Received: 29 December 2017; Accepted: 9 April 2018

\begin{abstract}
Objectives: There is almost no data on antidepressant prescribing in older adults treated with polypharmacy, although this population represents approximately 50\% of older patients. These patients are frequently excluded from double-blind randomized controlled trials, meta-analyses and existing treatment guidelines. The main aim of this paper was to identify data on antidepressant prescribing in depressed older adults on polypharmacy using a systematic review.

Methods: Randomized controlled clinical trials (RCTs) and other clinical trials in Medline/PubMed without language limitation (-2017) were searched to identify those with older depressed patients on polypharmacy. Only elderly patients (>65 years as mean) were included. Only approved antidepressants were included.

Results: The systematic search identified 26 different clinical trials, although only one clinical open label trial with sertraline met the final inclusion criteria. This sertraline trial indicated the absence of clinically important drug-drug interactions and confirmed the effectiveness and safety of sertraline in routine clinical practice. Heterogeneity in this trial was high in almost all the categories except attrition and reporting bias.

Conclusions: Sertraline has the highest evidence level in older adults with depression on polypharmacy. According to the results of this review and due to a low number of appropriate trials, a basic understanding of psychopharmacology is the possible approach to avoid serious problematic drug combinations in these patients. Newer RCTs are also urgently needed. This is the first systematic review including patients treated with polypharmacy, and therefore, its results are important in the field of evidence-based medicine.
\end{abstract}

\section{Keywords}

Antidepressants, older patients, medication prescribing, polypharmacy, systematic review, sertraline.

\section{INTRODUCTION}

Clinical depression (e.g., major depressive disorder) is common in older adults. Its prevalence in the community is around $15 \%$, and milder forms of depression are more common. The number of people over the age of 60 years is expected to double by 2050 , and hence, interventions for this often long-term and recurrent condition are increasingly important (Wilkinson and Izmeth 2016). In a meta-analysis, the authors found that antidepressants (tricyclic, selective serotonin-reuptake inhibitors, and a mixed group of other drugs) were significantly better than placebo, with an average reduction in symptom severity of $48.0 \%$ versus $31.3 \%$ (Gerson et al. 1999). Based on drug-to-drug comparisons, the selective serotonin-reuptake inhibitors appeared to be as effective as heterocyclic drugs (McCusker et al. 1998). In real clinical practice, often one antidepressant may seem to be more effective than another, although there is little advantage for one antidepressant class over another that is supported by the literature. In another meta-analysis, the authors compared different antidepressant groups in depressed older patients. They found that older patients showed no differences in antidepressant class outcomes in terms of efficacy and tolerability (Mittmann et al. 1997). Although antidepressant treatment efficacy has been shown, compared with younger individuals, older patients may be more likely to experience relapses and less likely to achieve a full response to treatment with antidepressant medications (Dew et al. 2007, Driscoll et al. 2005, Driscoll et al. 2007). Although these results are important, patients treated with polypharmacy have not been included in these trials, which means that most patients in 'real clinical practice' are not included in these important papers; hence, an evidence-based approach based on welldesigned network meta-analyses and clinical trials should be used to inform the choice of pharmacotherapy in this population. 
Polypharmacy is a world-wide problem and is often considered in a negative way in relation to good clinical practice although many patients indeed need many of their medications. Four out of 5 people aged 75 years and above are taking medications, and $36 \%$ receive 4 or more medications at the same time, which means that most patients in this population are not considered in the existing treatment guidelines (Quality and Outwork framework 2012). The probability of a drug-drug interaction (DDI) increases with the number of medications (polypharmacy). Specifically, a patient taking 5-9 medications had a 50\% probability, whereas the risk increased to $100 \%$ when a patient was found to be taking 20 or more medications (Doan et al. 2013). The probability of a DDI increased with the number of medications. Addition of each medication to a 5-drug regimen conferred a $12 \%$ increased risk of a potential CYPmediated DDI (Doan et al. 2013). In terms of polypharmacy, antidepressants are often prescribed inappropriately, as was shown in an Austrian cross-sectional study that included 48 out of 50 nursing homes and 1,844 out of 2,005 residents. The authors found high prevalence of potentially inappropriate prescriptions (PIPs). The prevalence of residents with at least one PIP was 70.3\% (95\% CI 67.2-73.4) and the antidepressants amitriptyline and maprotiline as PIM were prescribed very often (Mann et al. 2013).

In addition, most older depressed adults are excluded from the existing treatment guidelines and well-designed RCTs and meta-analyses, which means that there is a great lack of evidence to manage these patients in real clinical practice. Thus, the main aim of this paper was to identify the data on antidepressant treatment in depressed older adults on polypharmacy using a systematic review approach.

\section{METHODS}

\section{Search Strategy}

Preferred Reporting Items for Systematic Reviews and MetaAnalyses (PRISMA) guidelines-review protocol was used to conduct this review and this is summarized in detail in the Appendix 1 (Shamseer et al. 2015). A systematic review in Medline/Pubmed (-1.12.2017) was conducted with the following search strategy limited to human studies: polypharmacy AND antidepressants. In addition, references from meta-analyses on this topic were checked. Congress abstracts were not included if they were not published as regular articles in Medline/Pubmed. A literature search protocol is presented as a flow chart (Figure 1). A filter for
RCTs and clinical trials was applied when searching in Medline/Pubmed.

\section{Inclusion/exclusion criteria}

For inclusion in the systematic review, studies had to meet predefined PICOS + E requirements: specified population, intervention, comparator(s), outcome(s), study design, and exclusion criteria, for study inclusion (Shamseer et al. 2015). Through the searching process, only trials with older adults (65 years or more as mean) were included. Polypharmacy (e.g., more than one medication in addition to an antidepressant in the trial) was also checked in all the trials and when polypharmacy was not described, the trials were excluded. All types of polypharmacy were included. Trials including patients with any type of indication for antidepressant were included, although only papers with depression as the indication for treatment with antidepressants were searched in more detail. Trials with treatment-resistant depression and trials with 2 antidepressants concomitantly were excluded. The results were not divided according to the dosage regimen or type of depression. Trials with unclear methodology were also excluded (e.g., no treatment efficacy measurement, no discontinuation report, etc.). Besides the RCTs, only trials found within the clinical trials' filter were included.

\section{Study selection, data extraction and outcomes}

MS and JSM searched the trials with the defined searching strategy. MS identified appropriate trials. We extracted the following from each trial: title of the paper (full title); name of the authors; publication year; study design (double blind; single blind; open label); inclusion/exclusion criteria; interventions; outcomes (primary and secondary); main conclusions. In those trials where more outcomes were reported, a hierarchy was made by MS. Only the primary outcomes were identified and used for this review. To assess the risk of bias, the Cochrane RoB 1.0 tool was used to determine different sources of bias, that is, random sequence generation, allocation concealment, blinding of participants and personnel, blinding of outcome assessment, selective reporting, and other biases (Higgins et al. 2011). The results of the bias were presented in the table form. The two reviewers (JSM and MS) independently assessed the risk of bias. A pairwise or network meta-analysis was not performed. This systematic review was conducted without financial or other support. 


\section{RESULTS}

\section{Search results}

In total, 26 different clinical trials were found in Medline/ Pubmed, of which 12 were RCTs. From all the 26 trials that were conducted, only one open label noncomparative multicentre trial was appropriate to include in the final analysis (Arranz et al. 1997). Other trials were not appropriate because they didn't include antidepressants and/or elderly patients and/or polypharmacy. A lack of polypharmacy was the most important criteria for exclusion. Detailed results are presented in Figure 1 (flow chart). When we searched with only search strategy antidepressants, we obtained 16330 papers and when the search was carried out with only polypharmacy we obtained 307 papers.

\section{Clinical outcomes and risk of bias}

We found only one appropriate trial (Arranz et al. 1997). In this study, a noncomparative, observational, multicentre study over 8 weeks was conducted to assess the effectiveness and tolerability of sertraline (50-200 mg/day) in 1437 elderly depressed outpatients with a mean (S.D.) age of 68 (6.3) years (range 60-92) in a routine clinical practice. Depressive symptoms were monitored using the Montgomery-Åsberg Depression Rating Scale (MADRS) at baseline and at weeks 2, 4, 6 and 8 . The mean dose of sertraline at the final visit was $85.2 \mathrm{mg} /$ day (48\% of patients were given the initial dose throughout the study). At the end of the study, the mean percentage change of MADRS score from baseline was $61 \%$ $(\mathrm{P}<0.001) . \mathrm{A}>$ or $=50 \%$ decrease in MADRS score was obtained in $70 \%$ of patients. Sertraline was well tolerated. Side effects occurred in $23 \%$ of patients, although only $5.1 \%$ withdrew because of adverse events. There were no significant differences in the antidepressant effectiveness or occurrence of side effects when patients with and without concomitant pathologic conditions or with and without concurrent medications were compared (total of $68 \%$ of patients received concurrent medications). No concurrent antidepressant and serotoninergic medications were permitted during the study, although benzodiazepine co-medication for treating anxiety associated with depression was allowed. Benzodiazepines and cardiovascular drugs (antihypertensives, calcium channel blockers, cardiotonics, etc.) were the most frequently prescribed medicines. These findings suggested the absence of clinically important DDIs and confirmed the effectiveness and safety of sertraline in routine clinical practice for treating older depressed outpatients (Arranz et al. 1997). These results show that polypharmacy (defined as more than one

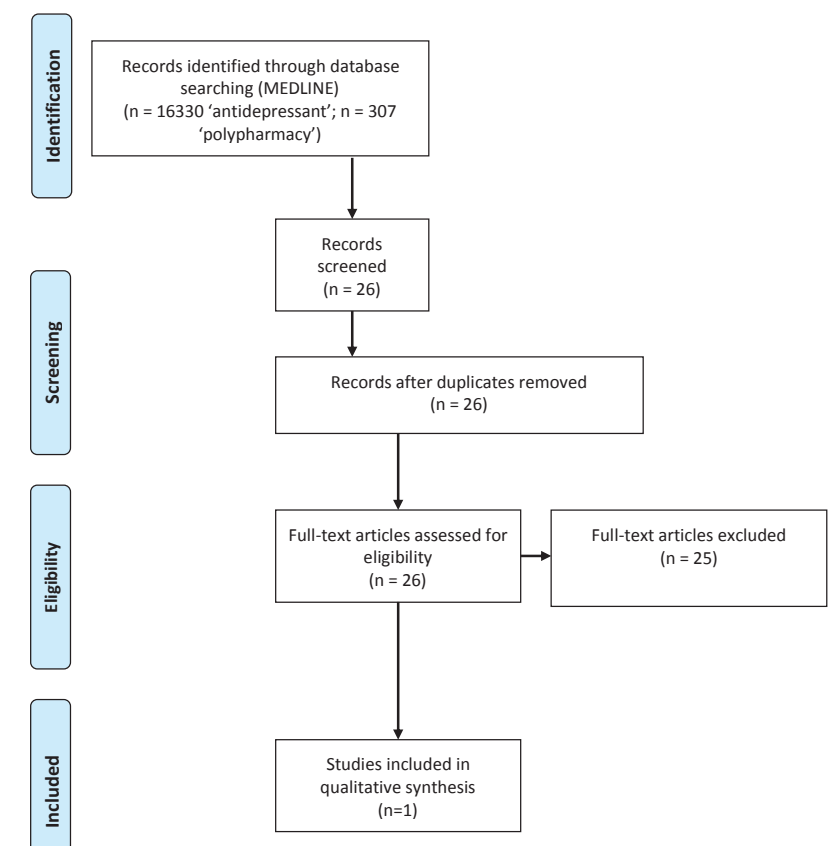

Figure 1. A flow chart of systematic review.

medication concomitantly used) had no impact on the final treatment's effectiveness and adverse events, which suggests that sertraline had a low potential for significant DDIs in real clinical practice.

This trial was found to have an overall high risk of bias because of its methodology (open label non-comparative multicentre trial), and almost all categories (outcomes blinding) were a source of bias, except attrition and reporting bias. Selection, performance, and detecting biases were high, because this trial was not a double blind, randomized, controlled trial (Table 1).

\section{DISCUSSION}

Results of this systematic review show that there are almost no trials in this patient population, as we only found one trial that met the inclusion criteria. The trial found that sertraline has the highest evidence for its use in older adults on polypharmacy, primarily because of its very low impact on potential DDIs as a consequence of polypharmacy (Arranz et al. 1997). These results are in line with a network meta-analysis published by Cipriani et al. (Cipriani et al. 2009), where sertraline was the best choice when starting a treatment for moderate to severe major depression in adults because it had the most favourable balance between benefits, acceptability, and acquisition cost. Although this network meta-analysis excluded older adult 
Table 1. Presentation of risk of bias assessments for trials included in this systematic review. Low risk of bias (+), high risk of bias (-), unclear risk of bias (?).

\begin{tabular}{|c|c|c|c|c|c|c|c|c|}
\hline & & \multicolumn{2}{|c|}{ Selection bias } & $\begin{array}{c}\text { Performance } \\
\text { bias }\end{array}$ & $\begin{array}{c}\text { Detection } \\
\text { bias }\end{array}$ & $\begin{array}{c}\text { Attrition } \\
\text { bias }\end{array}$ & $\begin{array}{c}\text { Reporting } \\
\text { bias }\end{array}$ & \\
\hline Study & Drug tested & $\begin{array}{c}\text { Random } \\
\text { sequence } \\
\text { generation }\end{array}$ & $\begin{array}{c}\text { Allocation } \\
\text { concealment }\end{array}$ & $\begin{array}{l}\text { Blinding of } \\
\text { participants and } \\
\text { personnel }\end{array}$ & $\begin{array}{l}\text { Blinding of } \\
\text { outcome } \\
\text { assessment }\end{array}$ & $\begin{array}{l}\text { Incomplete } \\
\text { outcome } \\
\text { data }\end{array}$ & $\begin{array}{l}\text { Selective } \\
\text { reporting }\end{array}$ & $\begin{array}{c}\text { Other } \\
\text { bias }\end{array}$ \\
\hline Aranz, 1997 & Sertraline & - & - & - & - & + & + & $?$ \\
\hline
\end{tabular}

patients on polypharmacy, results are comparable with those of our systematic review, which means that sertraline in this population has been supported by the highest evidence level obtained from a well-designed meta-analysis and a more 'real clinical practice' systematic review. These results show that there is some evidence to support the use of sertraline in this population, although this evidence is very weak, because of the fact that long-term treated patients and different types of polypharmacy were not included in these reviews. Thus, welldesigned long term head-to-head trials should be conducted to obtain more relevant results, and these should be followed by a network meta-analysis of these trials. These results could be connected with a network meta-analysis of older adults (60 years and older) where for partial response, sertraline $(\mathrm{RR}=1.28)$, paroxetine $(\mathrm{RR}=1.48)$, and duloxetine $(\mathrm{RR}=$ 1.62) were significantly better than placebo (Thorlund et al. 2015). The remaining interventions yielded RRs lower than 1.20. For dizziness, duloxetine $(R R=3.18)$ and venlafaxine $(\mathrm{RR}=2.94)$ were statistically significantly worse than placebo. Compared with placebo, sertraline had the lowest RR for dizziness (1.14) and fluoxetine being the second lowest (1.31). Citalopram, escitalopram, and paroxetine, all had RRs between 1.4 and 1.7. These results are in line with our results, that sertraline has the best risk-benefit ratio, although there was no data about polypharmacy in the included trials (Thorlund et al. 2015). In addition to this weak evidence in this important population, a solid knowledge of basic clinical pharmacology in pharmacotherapy planning in older adults would be the best approach to avoid serious DDIs and adverse events as was shown in various important papers (Holt et al. 2010, American Geriatrics Society 2015, O’Mahony et al. 2015). The use of sertraline in patients on polypharmacy could be explained pharmacologically. Sertraline has a low potential for DDIs because it is not a strong inductor nor inhibitor of cytochrome 450 isoforms. According to in vitro studies, sertraline is metabolized by multiple cytochrome 450 isoforms: CYP2D6, CYP2C9, CYP2B6, CYP2C19 and CYP3A4. It is important to add that there are few possibilities that the inhibition of any single isoform could cause clinically significant changes in the pharmacokinetics of sertraline (Obach et al. 2005). These important pharmacokinetic properties of sertraline show that it has a small number of possible clinically important DDIs in real clinical practice. Despite the fact that these pharmacokinetic properties are important, it is clear that sertraline cannot be used in all older patients on polypharmacy because antidepressants have different mechanisms of action that are very important when prescribing. These important issues are often excluded from clinical trials, in spite of their importance in daily practice. It is also important to add that sertraline has also been involved in some important DDIs in elderly patients. These mostly included DDIs via the CYP P450 3A4 system, where strong inhibitors/inductors could have an impact on sertraline adverse events or lack of efficacy (Khan et al. 2000). Thus, in addition to the results of this systematic review, a pharmacotherapy review before prescribing should be an important option in order to avoid serious DDIs and treatment failure in this population.

We should emphasise that in addition to the number of potential DDIs, the type of DDI is also very important for pharmacotherapy planning. Pharmacodynamic DDIs (e.g., QTc prolongation) are often associated with antidepressant use, especially in case reports (e.g., citalopram, duloxetine, venlafaxine, andbupropion), although these effectsareexcluded from treatment guidelines and often do not include older patients on polypharmacy (American Psychiatric Association 2010, Jasiak and Bostwick 2014, Štuhec 2013, Tampi et al. 2015). This has not been observed in a network meta-analysis in which mirtazapine, escitalopram, venlafaxine, and sertraline were significantly more efficacious than duloxetine (odds ratios [OR] 1.39, 1.33, 1.30 and 1.27, respectively), fluoxetine $(1.37,1.32,1.28$, and 1.25 , respectively), fluvoxamine (1.41, $1.35,1.30$, and 1.27 , respectively), paroxetine $(1.35,1.30$, 1.27 , and 1.22 , respectively), and reboxetine $(2.03,1.95,1.89$, and 1.85 , respectively). Escitalopram and sertraline showed the best profile of acceptability, leading to significantly fewer discontinuations than did duloxetine, fluvoxamine, paroxetine, reboxetine, and venlafaxine (Cipriani et al. 2009). Although both meta-analyses are interesting, there is no data on polypharmacy use including antidepressants, and therefore, these meta-analyses including the famous Sequenced 
Treatment Alternatives to Relieve Depression $\left(\mathrm{STAR}^{\star} \mathrm{D}\right)$ trial are not representative for this patient population (Cipriani et al. 2009, Sinyor et al. 2010).

Although there is plenty of evidence described for the general population, there is a lack of supportive trials in older patients treated with polypharmacy in relation to the choice of antidepressant (no RCTs). A cautious psychopharmacological approach is necessary when prescribing in order to avoid serious adverse events, DDIs, and lack of treatment efficacy. Although these patients are often treated in real clinical practice they are actually not covered completely (or even not covered at all) in available treatment guidelines. This means that practitioners should use a cautious psychopharmacological approach in this patient group. One possible approach to reduce adverse events in this population is to use different lists of inappropriate medications (e.g., the STOPP/START criteria, 2015; the American Geriatrics Society Beers Criteria for Potentially Inappropriate Medication Use in Older Adults, the PRISCUS list), which would help minimising the use of inappropriate antidepressants in this population (American Geriatrics Society 2015, Holt et al. 2010, O’Mahony et al. 2015). According to the PRISCUS criteria fluoxetine, the MAO inhibitor tranylcypromine, and TCAs including amitriptyline, doxepine, imipramine, clomipramine, maprotiline and trimipramine, should be avoided in this population. Trazodone, other SSRIs (e.g., escitalopram, sertraline), and mirtazapine could be used. A reduced dose of TCAs is recommended for elderly patients because elderly patients taking TCAs have been reported to have a higher frequency of confusion and other manifestations relating to the central nervous system (Holt et al. 2010). However, although the PRISCUS list is useful in daily practice, it has many limitations. The PRISCUS list for the elderly was developed specifically for use in Germany, which means that many medications are not included (i.e., those which are not available in Germany). Another important limitation is the lack of comparative evidence for each medication group, which is not the case in the 2015 American Geriatrics Society Beers Criteria for Potentially Inappropriate Medication Use in Older Adults (American Geriatrics Society 2015). In the Beers criteria, a strength of recommendation has been added, which helps clinicians and clinical pharmacists to categorise different medication groups within the list. Amitriptyline, amoxapine, clomipramine, desipramine, doxepin $>6 \mathrm{mg} / \mathrm{d}$, imipramine, nortriptyline, paroxetine, protriptyline, and trimipramine have been included in the Beers criteria 2015 with a high quality of evidence and a strong recommendation to avoid in clinical practice in this population (American Geriatrics Society 2015).
This systematic review also has many important limitations. We also included non RCTs, which increased the bias, although these trials are closer to the real clinical patients. It also only included patients on polypharmacy, which could be defined differently in different countries and we included only those trials with antidepressants, although other medications are also used for depression treatment in older adults (e.g., antipsychotics). Another very important limitation is the lack of long term trials because patients treated with antidepressants are treated for a long period of time (e.g., 12 months). Another limitation is the lack of comparative polypharmacy trials because different medications can have a different impact on DDIs. In addition, patients' habits are also very important (e.g., smoking), as they can have an impact on drug metabolism (e.g., clozapine, duloxetine) (Mangerud et al. 2014). The next limitation is the lack of dose comparison. Some adverse events could be dose-dependent. Antidepressants also have different half-lives, which means that short-term trials including medications with long halflife including polypharmacy can lead to inappropriate results because many DDIs can occur after many weeks of treatment (e.g., DDIs with fluoxetine). Despite these limitations, this is the first systematic review including patients treated with polypharmacy without treatment-resistant depression, and therefore, the results are important in the field of evidencebased medicine.

\section{CONCLUSION}

Sertraline has the highest level of evidence for use in older adults with depression and on polypharmacy. An individual treatment approach including different PIM lists and basic pharmacology considerations is one of the possible tools to manage these patients. More 'real clinical setting' trials and RCTs are needed to provide further evidence on the important topic of optimisation of the treatment of depression in older patients on polypharmacy.

\section{ACKNOWLEDGEMENTS}

None. 


\section{REFERENCES}

American Geriatrics Society 2015 Beers Criteria Update Expert Panel: American Geriatrics Society 2015 Updated Beers Criteria for Potentially Inappropriate Medication Use in Older Adults. J Am Geriatr Soc. 2015; 63: 2227-2246.

Arranz FJ, Ros S. Effects of comorbidity and polypharmacy on the clinical usefulness of sertraline in elderly depressed patients: an open multicentre study. J Affect Disord. 1997; 46: 285-91.

Cipriani A, Furukawa TA, Salanti G, Geddes JR, Higgins JP, Churchill R, Watanabe N, Nakagawa A, Omori IM, McGuire H, Tansella M, Barbui C. Comparative efficacy and acceptability of 12 new-generation antidepressants: a multiple-treatments metaanalysis. Lancet. 2009; 373: 746-58.

Dew MA, Whyte EM, Lenze EJ, Houck PR, Mulsant BH, Pollock BG, Stack JA, Bensasi S, Reynolds CF. Recovery from major depression in older adults receiving augmentation of antidepressant pharmacotherapy. Am J Psychiatry. 2007; 164: 892-899

Doan J, Zakrzewski-Jakubiak H, Roy J, Turgeon J, Tannenbaum C. Prevalence and risk of potential cytochrome P450-mediated drugdrug interactions in older hospitalized patients with polypharmacy. Ann Pharmacother. 2013; 47: 324-332

Driscoll HC, Basinski J, Mulsant BH, Butters MA, Dew MA, Houck PR, Mazumdar S, Miller MD, Pollock BG, Stack JA, Schlernitzauer MA, Reynolds CF. Late-onset major depression: clinical and treatment-response variability. Int J Geriatr Psychiatry. 2005; 20: 661-667

Driscoll HC, Karp JF, Dew MA, Reynolds CF. Getting better, getting well: understanding and managing partial and non-response to pharmacological treatment of non-psychotic major depression in old age. Drugs Aging. 2007; 24: 801-814

Gerson S, Belin TR, Kaufman A, Mintz J, Jarvik L. Pharmacological and psychological treatments for depressed older patients: a metaanalysis and overview of recent findings. Harv Rev Psychiatry. 1999; 7: 1-28.

Higgins JPT, Altman DG, Stearne JAC (editors). Assessing risk of bias in included studies. In: Higgins JPT, Green S (editors). Cochrane Handbook for Systematic Reviews of Interventions Version 5.1.0 (updated March 2011). The Cochrane Collaboration (2011) www.cochrane-handbook.org. Accessed, March 2017.
Holt S, Schmiedl S, Thürmann PA. Potentially inappropriate medications in the elderly: the PRISCUS list. Dtsch Arztebl Int. 2010; 107: 543-51.

Jasiak NM, Bostwick JR. Risk of QT/QTc prolongation among newer non-SSRI antidepressants. Ann Pharmacother. 2014; 48: 1620-8.

Khan A, Shad MU, Preskorn SH. Lack of sertraline efficacy probably due to an interaction with carbamazepine. J Clin Psychiatry. 2000; 61: 526-7.

Mangerud WL, Bjerkeset O, Holmen TL, Lydersen S, Indredavik MS. Smoking, alcohol consumption, and drug use among adolescents with psychiatric disorders compared with a population based sample. J Adolesc. 2014; 37: 1189-1199.

Mann E, Haastert B, Böhmdorfer B, Frühwald T, Iglseder B, Roller-Wirnsberger R, Meyer G. Prevalence and associations of potentially inappropriate prescriptions in Austrian nursing home residents: secondary analysis of a cross-sectional study. Wien Klin Wochenschr. 2013; 125: 180-188.

McCusker J, Cole M, Keller E, Bellavance F, Berard A. Effectiveness of treatments of depression in older ambulatory patients. Arch Intern Med. 1998; 13; 158: 705-12.

Mittmann N, Herrmann N, Einarson TR, Busto UE, Lanctôt KL, Liu BA, Shulman KI, Silver IL, Narango CA, Shear NH. The efficacy, safety and tolerability of antidepressants in late life depression: a meta-analysis. J Affect Disord. 1997; 46: 191-217.

Obach RS, Cox LM, Tremaine LM. Sertraline is metabolized by multiple cytochrome P450 enzymes, monoamine oxidases, and glucuronyl transferases in human: an in vitro study. Drug Metab Dispos. 2005; 33: 262-70.

O'Mahony D, O'Sullivan D, Byrne S, O'Connor MN, Ryan C, Gallagher P. STOPP/START criteria for potentially inappropriate prescribing in older people: version 2. Age Ageing. 2015; 44: 213-8.

Practice guideline for the Treatment of Patients with Major Depressive Disorder, Third Edition, APA. American Psychiatric Association (APA) treatment guidelines (2010). Available from: https://psychiatryonline.org/pb/assets/raw/sitewide/practice_ guidelines/guidelines/mdd.pdf 
Quality and Outwork framework 2012 (Accessed on 27 of January 2018): http://www.nhsemployers.org/Aboutus/Publications/ Documents/QOF_2012-13.pdf

Shamseer L, Moher D, Clarke M, Ghersi D, Liberati A, Petticrew M, Shekelle P, Stewart LA; PRISMA-P Group. Preferred reporting items for systematic review and meta-analysis protocols (PRISMA-P) 2015: elaboration and explanation. BMJ. 2015; 349: g7647.

Sinyor M, Schaffer A, Levitt A. The sequenced treatment alternatives to relieve depression (STAR*D) trial: a review. Can J Psychiatry. 2010; 55: 126-35.

Štuhec M. Duloxetine-induced life-threatening long QT syndrome. Wien Klin Wochenschr. 2013; 125: 165-6
Tampi RR, Balderas M, Carter KV, Tampi DJ, Moca M, Knudsen A, May J. Citalopram, QTc Prolongation, and Torsades de Pointes. Psychosomatics. 2015; 56: 36-43.

Thorlund K, Druyts E, Wu P, Balijepalli C, Keohane D, Mills E. Comparative efficacy and safety of selective serotonin reuptake inhibitors and serotonin-norepinephrine reuptake inhibitors in older adults: a network meta-analysis. J Am Geriatr Soc. 2015; 63: 1002-9.

Wilkinson P, Izmeth Z. Continuation and maintenance treatments for depression in older people. Cochrane Database Syst Rev. 2016: 9: CD006727.

Appendix Table 1: PRISMA-P (Preferred Reporting Items for Systematic review and Meta-Analysis Protocols) 2015 checklist: recommended items to address in a systematic review protocol*

\begin{tabular}{|c|c|c|c|}
\hline $\begin{array}{l}\text { Section and } \\
\text { topic }\end{array}$ & $\begin{array}{l}\text { Item } \\
\text { No }\end{array}$ & Checklist item & $\begin{array}{l}\text { Reported on page \# } \\
\text { Title page not count }\end{array}$ \\
\hline \multicolumn{4}{|c|}{ ADMINISTRATIVE INFORMATION } \\
\hline \multicolumn{4}{|l|}{ Title: } \\
\hline Identification & $1 \mathrm{a}$ & Identify the report as a protocol of a systematic review & 1 \\
\hline Update & $1 b$ & If the protocol is for an update of a previous systematic review, identify as such & 1 \\
\hline Registration & 2 & $\begin{array}{l}\text { If registered, provide the name of the registry (such as PROSPERO) and } \\
\text { registration number }\end{array}$ & NA \\
\hline \multicolumn{4}{|l|}{ Authors: } \\
\hline Contact & 3a & $\begin{array}{l}\text { Provide name, institutional affiliation, e-mail address of all protocol authors; } \\
\text { provide physical mailing address of the corresponding author }\end{array}$ & Separate page \\
\hline Contributions & $3 b$ & Describe contributions of protocol authors and identify the guarantor of the review & Separate page \\
\hline Amendments & 4 & $\begin{array}{l}\text { If the protocol represents an amendment of a previously completed or published } \\
\text { protocol, identify as such and list changes; otherwise, state plan for documenting } \\
\text { important protocol amendments }\end{array}$ & NA \\
\hline \multicolumn{4}{|l|}{ Support: } \\
\hline Sources & $5 a$ & Indicate sources of financial or other support for the review & 2 \\
\hline Sponsor & $5 b$ & Provide name for the review funder and/or sponsor & NA \\
\hline $\begin{array}{c}\text { Role of sponsor } \\
\text { or funder }\end{array}$ & $5 c$ & $\begin{array}{l}\text { Describe roles of funder(s), sponsor(s), and/or institution(s), if any, in developing } \\
\text { the protocol }\end{array}$ & NA \\
\hline \multicolumn{4}{|c|}{ INTRODUCTION } \\
\hline Rationale & 6 & Describe the rationale for the review in the context of what is already known & 1 \\
\hline Objectives & 7 & $\begin{array}{l}\text { Provide an explicit statement of the question(s) the review will address with } \\
\text { reference to participants, interventions, comparators, and outcomes (PICO) }\end{array}$ & 2 \\
\hline \multicolumn{4}{|c|}{ METHODS } \\
\hline Eligibility criteria & 8 & $\begin{array}{c}\text { Specify the study characteristics (such as PICO, study design, setting, time frame) } \\
\text { and report characteristics (such as years considered, language, publication status) } \\
\text { to be used as criteria for eligibility for the review }\end{array}$ & 2 \\
\hline $\begin{array}{l}\text { Information } \\
\text { sources }\end{array}$ & 9 & $\begin{array}{c}\text { Describe all intended information sources (such as electronic databases, contact } \\
\text { with study authors, trial registers or other grey literature sources) with planned } \\
\text { dates of coverage }\end{array}$ & 2 \\
\hline Search strategy & 10 & $\begin{array}{l}\text { Present draft of search strategy to be used for at least one electronic database, } \\
\text { including planned limits, such that it could be repeated }\end{array}$ & 2 \\
\hline
\end{tabular}


Continued Appendix Table 1: PRISMA-P (Preferred Reporting Items for Systematic review and Meta-Analysis Protocols) 2015 checklist: recommended items to address in a systematic review protocol*

\begin{tabular}{|c|c|c|c|}
\hline $\begin{array}{l}\text { Section and } \\
\text { topic }\end{array}$ & $\begin{array}{l}\text { Item } \\
\text { No }\end{array}$ & Checklist item & $\begin{array}{l}\text { Reported on page \# } \\
\text { Title page not count }\end{array}$ \\
\hline \multicolumn{4}{|l|}{ Study records: } \\
\hline $\begin{array}{c}\text { Data } \\
\text { management }\end{array}$ & $11 a$ & $\begin{array}{l}\text { Describe the mechanism(s) that will be used to manage records and data } \\
\text { throughout the review }\end{array}$ & 2 \\
\hline $\begin{array}{l}\text { Selection } \\
\text { process }\end{array}$ & $11 b$ & $\begin{array}{l}\text { State the process that will be used for selecting studies (such as two independent } \\
\text { reviewers) through each phase of the review (that is, screening, eligibility and } \\
\text { inclusion in meta-analysis) }\end{array}$ & 2 \\
\hline $\begin{array}{l}\text { Data collection } \\
\text { process }\end{array}$ & $11 \mathrm{c}$ & $\begin{array}{l}\text { Describe planned method of extracting data from reports (such as piloting forms, } \\
\text { done independently, in duplicate), any processes for obtaining and confirming data } \\
\text { from investigators }\end{array}$ & 2 \\
\hline Data items & 12 & $\begin{array}{l}\text { List and define all variables for which data will be sought (such as PICO items, } \\
\text { funding sources), any pre-planned data assumptions and simplifications }\end{array}$ & 2 \\
\hline $\begin{array}{l}\text { Outcomes and } \\
\text { prioritization }\end{array}$ & 13 & $\begin{array}{l}\text { List and define all outcomes for which data will be sought, including prioritization } \\
\text { of main and additional outcomes, with rationale }\end{array}$ & 2 \\
\hline $\begin{array}{l}\text { Risk of bias } \\
\text { in individual } \\
\text { studies }\end{array}$ & 14 & $\begin{array}{c}\text { Describe anticipated methods for assessing risk of bias of individual studies, } \\
\text { including whether this will be done at the outcome or study level, or both; state } \\
\text { how this information will be used in data synthesis }\end{array}$ & 2 \\
\hline \multirow{4}{*}{ Data synthesis } & $15 a$ & Describe criteria under which study data will be quantitatively synthesised & 2 \\
\hline & $15 b$ & $\begin{array}{l}\text { If data are appropriate for quantitative synthesis, describe planned summary } \\
\text { measures, methods of handling data and methods of combining data from studies, } \\
\text { including any planned exploration of consistency (such as }\left.\right|^{2} \text {, Kendall's } \mathrm{T} \text { ) }\end{array}$ & NA \\
\hline & $15 c$ & $\begin{array}{c}\text { Describe any proposed additional analyses (such as sensitivity or subgroup } \\
\text { analyses, meta-regression) }\end{array}$ & NA \\
\hline & $15 d$ & If quantitative synthesis is not appropriate, describe the type of summary planned & NA \\
\hline Meta-bias(es) & 16 & $\begin{array}{c}\text { Specify any planned assessment of meta-bias(es) (such as publication bias across } \\
\text { studies, selective reporting within studies) }\end{array}$ & NA \\
\hline $\begin{array}{l}\text { Confidence } \\
\text { in cumulative } \\
\text { evidence }\end{array}$ & 17 & $\begin{array}{l}\text { Describe how the strength of the body of evidence will be assessed (such as } \\
\text { GRADE) }\end{array}$ & NA \\
\hline
\end{tabular}

* It is strongly recommended that this checklist be read in conjunction with the PRISMA-P Explanation and Elaboration (cite when available) for important clarification on the items. Amendments to a review protocol should be tracked and dated. The copyright for PRISMA-P (including checklist) is held by the PRISMA-P Group and is distributed under a Creative Commons Attribution Licence 4.0.

From: Shamseer L, Moher D, Clarke M, Ghersi D, Liberati A, Petticrew M, Shekelle P, Stewart L, PRISMA-P Group. Preferred reporting items for systematic review and meta-analysis protocols (PRISMA-P) 2015: elaboration and explanation. BMJ. 2015 Jan 2;349ljan02 1):g7647. 